\title{
O TESTE DE PFISTER E SUA CONTRIBUIÇÃO PARA DIAGNÓSTICO DA ESQUIZOFRENIA
}

\author{
Anna Elisa de Villemor Amaral ${ }^{\star}$ \\ Ricardo Primi ${ }^{\star \star}$ \\ Renata da Rocha Campos Franco $\star \star \star ~$ \\ Flávia Helena Zanetti Farah ${ }^{\star \star \star \star}$

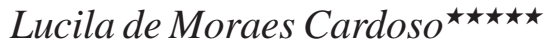 \\ Telma Claudina da Silva
}

\begin{abstract}
Resumo
Procurou-se identificar indicadores no teste das Pirâmides Coloridas de Pfister que pudessem auxiliar nos diagnósticos de pacientes esquizofrênicos. Tal iniciativa justifica-se pela escassez de pesquisas de validade desse teste para diagnóstico em psicopatologia. Os resultados obtidos demonstraram que a execução de tapetes furados e desequilibrados constitui um indicador significativo de esquizofrenia, principalmente quando associado a não execução de formações em camadas multicromáticas e a constância absoluta das cores vermelha e marrom. Não foram identificadas alterações significativas na freqüência da cor branca conforme previsto em outros estudos. Os resultados demonstram que a sensibilidade do Pfister para diagnóstico de esquizofrenia, com base nesses indicadores, é relativa devido à possibilidade de falsos negativos e falsos positivos, podendo, entretanto contribuir positivamente para a avaliação psicológica quando associado com outras técnicas. Esse estudo vem ao encontro da necessidade de verificar os alcances e as limitações do uso de testes psicológicos.
\end{abstract}

Palavras-chave: Teste das Pirâmides Coloridas de Pfister. Avaliação Psicológica. Psicodiagnóstico. Esquizofrenia. Psicopatologia.

^ Professora associada. Doutora no Programa de estudos Pós-graduados em Psicologia da Universidade São Francisco.

E-mail: Anna.villemor@saofrancisco.edu.br

$\star \star$ Professor associado. Doutor no Programa de estudos Pós-graduados em Psicologia da Universidade São Francisco.

$\star \star \star$ Bolsista Fapesp de iniciação científica da Universidade São Francisco.

$\star \star \star \star$ Mestranda no Programa de estudos Pós-graduados em Psicologia da Universidade São Francisco.

$\star \star \star \star \star$ Bolsista Fapesp de iniciação científica da Universidade São Francisco.

$\star \star \star \star \star \star$ Bolsista PIBIC da Universidade São Francisco. 


\title{
The pfister test's contribution to the assessment of schizophrenia
}

\begin{abstract}
The aim of this study is to identify the aspects in the Color Pyramid Test of Pfister that may be useful to the assessment of schizophrenic patients. It is justified due to the scarceness of studies on the validity of Pfister Test for the psychopathological assessment. The results demonstrate that the disorganized forms called holey rugs or unbalanced rugs are significant indicators of schizophrenia, especially when associated with the non execution of stratified forms or the absolute constancy of the colors red and brown. We didn't find significant alterations on the frequency of the white color as found on other studies. Our results indicate that the sensibility of the Pfister test for the diagnosis of schizophrenia is limited due to the possibility of false positives and false negatives, but it could help in the psychological assessment when associated to other techniques. This study indicates the necessity to verify the possibilities and limitations in the use of psychological tests.
\end{abstract}

Keywords: Color Pyramid Test Pfister. Psychological assessment. Schizophrenia. Psychopathology

\section{INTRODUÇÃo}

O Teste das Pirâmides Coloridas de Pfister (TPC) é um instrumento de avaliação psicológica bastante utilizado no meio clínico, acadêmico e de pesquisas, mas são raros os estudos normativos e de validade realizados com essa técnica. Mais raros ainda, são os estudos em psicopatologia. Essa pesquisa é decorrente da necessidade de verificar não apenas os indicadores do Pfister que possam auxiliar nos processos psicodiagnósticos, mas também o de rever a validade das interpretações atribuídas a vários índices e resultados obtidos com o teste.

Dentro de um estudo mais amplo realizado com vários grupos de pacientes e não pacientes, destacamos nesse trabalho os achados para um grupo de esquizofrênicos.

Se por um lado, a esquizofrenia é uma patologia bastante freqüente e seu diagnóstico pode ser feito por muitos instrumentos de valor comprovado, por outro lado, existem ocasiões em que o uso de instrumentos não-verbais, como é o caso do TPC, pode ser útil onde há comprometimento da expressão e comunicação verbal ou em casos de tentativa de simulação ou distorção de resultados obtidos com instrumentos que dependem do auto-relato, mais passíveis de controle e manipulação. Justifica-se, portanto, o desenvolvimento de instrumentos com ca- 
racterísticas variadas que facilitem o processo diagnóstico nas mais diversas circunstâncias e contribuam para um bom encaminhamento e tratamento.

O TPC é uma técnica com características bastante propícias para aceitação e realização da tarefa, uma vez que é de manejo simples, requer curto espaço de tempo, não depende da expressão verbal e é geralmente considerada lúdica. No caso do diagnóstico psicopatológico, embora não existam estudos de validade para o diagnóstico da esquizofrenia, encontramos na literatura considerações a respeito do aspecto formal da pirâmide e da maior ou menor incidência de certas cores como sendo características de um funcionamento psicótico e mais propriamente esquizofrênico. Assim, Villemor Amaral (1978) chama a atenção para os tapetes furados e desorganizados e para a alta incidência da cor branca como sendo características presentes nas produções de pacientes com frágil equilíbrio da personalidade como é o caso de psicóticos em geral nesse teste, e comenta sobre seu significado ligado à fragilidade estrutural e dissociação do curso do pensamento, "perda do contato com a realidade e perda do controle dos impulsos" (VILLEMOR AMARAL, 1978, p.108).

Justo e Van Kolck (1976) também atribuem ao branco aumentado o significado de estrutura de personalidade frágil sendo característico em esquizofrênicos. Esses autores citam pesquisas de O'Reilly (1957, apud JUSTO; VAN KOLCK, 1976) e Anzieu (1970, apud JUSTO; VAN KOLCK, 1976) que encontraram tapete rasgado em $77 \%$ de um grupo de esquizofrênicos. Entretanto citam também uma pesquisa de Beck (1964 apud JUSTO; VAN KOLCK, 1976) com 51 esquizofrênicos avaliados com o Pfister e com o MMPI, na qual não foi encontrada correlação significativa entre a escolha de cores e escalas desse inventário.

Heiss e Halder (1983) igualmente afirmam que o branco aparecia aumentado nos protocolos de esquizofrênicos e concordam com o fato de que tapetes denotam "fraqueza geral" (HEISS; HALDER, 1983, p.116) embora não associem essa forma como produção de esquizofênicos.

Carnio e Loureiro (1994) realizaram uma pesquisa com 20 esquizofrênicos e centraram sua análise no modo de execução, tempo de execução, fórmula cromática e na freqüência da cor branca e da síndrome de adaptação ao ambiente (S.A.A.) constituída pelas cores verde, azul e amarela. No que diz respeito às cores, encontraram uma diminuição do branco, contrariando as pesquisas expostas acima, e registram um aumento significativo de combinações da S.A.A. que denotam estreitamento e afastamento do real.

A esquizofrenia é uma patologia dentro do grupo das psicoses que se caracteriza fundamentalmente por alterações no nível do pensamento e da percepção decorrentes de um mau contato com a realidade. Embora existam subtipos da doença, as diferenças referem-se a variações sintomáticas, mantendo-se as limitações básicas no contato com a realidade e suas consequiências fundamentais.

Conforme o Manual Diagnóstico Estatístico de Transtornos Mentais (DSMIV), a esquizofrenia é uma perturbação com duração de pelo menos seis meses e com a ocorrência de pelo menos dois dos seguintes sintomas: delírio, alucinação, discurso desorganizado, comportamento desorganizado ou catatônico, 
embotamento do afeto e pouca produtividade do pensamento (alogia). Os diferentes subtipos constituem classificações baseadas na predominância de certos sintomas que têm sua utilidade principalmente para efeitos dos tratamentos medicamentosos uma vez que determinados sintomas reagem mais a certos medicamentos do que outros (GABBARD, 1998).

De acordo com a American Psychiatric Association (APA, 1995) sua prevalência no mundo é de 0,5 a $1,0 \%$, seu aparecimento costuma ser em torno da terceira década de vida e segundo Paim (1990) um diagnóstico precoce e um tratamento adequado podem modificar de forma favorável o curso da doença.

Para a psicanálise, a esquizofrenia tem sua origem na formação da estrutura da personalidade, nos primeiros meses de vida. Foi Melanie Klein (1982) quem trouxe contribuições bastante decisivas para a compreensão dessa patologia ao descrever a posição esquizo-paranóide e seus desdobramentos para a vida mental normal e patológica. Resumidamente, pode-se dizer que a experiência vital da amamentação constitui a primeira relação de objeto que traz tanto momentos de satisfação e prazer quanto momentos de frustração e angústias profundas relacionadas ao temor de aniquilamento. Por imaturidade do psiquismo do bebê, o objetomãe não é reconhecido como inteiro e as experiências de satisfação e de insatisfação são vividas como se fossem provocados por objetos distintos. As relações com o bom e o mau objeto são assim polarizadas, parciais, clivadas, sendo a cisão o principal mecanismo de defesa nessa fase, que tem como função preservar o objeto bom dos ataques dirigidos ao mau objeto. Uma má elaboração desse período impede a possibilidade de reconhecer o objeto como um ser total integrado e a relação mantém-se parcial. Nesse caso, o mecanismo de cisão pode se sobrepor, ser bastante intenso e predominante, impedindo que outros mecanismos de defesa mais evoluídos possam se desenvolver e seus efeitos passam a ser tão amplos que comprometem toda a percepção do indivíduo e suas relações com o mundo ao redor. No caso da esquizofrenia o que temos é justamente o predomínio de percepções e pensamentos cindidos e fragmentados que se estendem para a vida adulta afetando a estrutura da personalidade e todo seu funcionamento mental.

O objetivo deste trabalho foi o de verificar quais aspectos no teste apareceriam com maior frequiência entre os esquizofrênicos examinados de modo a distingui-los de um grupo de não pacientes, dando especial ênfase às características apontadas na literatura.

\section{MÉTOdo E PARTICIPANTES}

Foram sujeitos dessa pesquisa 20 pessoas com idade superior a 18 anos, de ambos os sexos e níveis de escolaridade e socioeconômico variados. Todos eram pacientes ligados a instituições de saúde mental de uma região do interior de São Paulo (hospital-dia ou internos em clínicas de atendimento psiquiátrico ou psicológico) que foram diagnosticados pela SCID-2000 como portadores da esquizofrenia e que estavam sendo medicados pelos psiquiatras responsáveis. 
Para comparação dos resultados obtidos com o grupo de pacientes tomouse como base os indicadores resultantes de uma investigação com fins normativos realizada com 110 indivíduos adultos não-pacientes residentes no interior de São Paulo, na mesma região em que foi coletada a amostra de pacientes.

\section{INSTRUMENTOS}

Para composição da amostra conforme critérios homogêneos foi utilizada a Entrevista Clínica Estruturada para o DSM-IV - SCID I (FIRST; SPITZER; GIBBON; WILLIAMS, 1996).

O Teste de Pfister foi utilizado na sua versão composta por um conjunto de três cartões em papel pardo com o esquema de uma pirâmide formada por 15 espaços quadrangulares e mais um jogo de quadrículos coloridos composto por dez cores subdivididas em 24 tonalidades. Conforme Villemor Amaral (1978) foram aplicadas somente às pirâmides bonitas. As cores que compõem o material do teste são o azul, verde e vermelho (quatro tonalidades de cada), violeta (três tonalidades), amarelo, laranja e marrom (duas tonalidades cada), preto, branco e cinza.

\section{Procedimento}

As instituições de saúde mental da região aceitaram colaborar com a pesquisa encaminhando os pacientes e oferecendo local para a realização do exame. As entrevistas estruturadas e o teste de Pfister foram aplicados nas instituições de atendimento dos pacientes, em local apropriado para um atendimento individual e sem interrupções. Cada paciente foi esclarecido sobre os propósitos da pesquisa e assinou termo de consentimento de participação.

O exame em todos os casos iniciou-se pela SCID para confirmação da inclusão do paciente na amostra seguindo critérios padronizados, utilizando-se da revisão geral e do questionário específico para esquizofrenia e transtornos psicóticos.

A seguir, solicitava-se a realização das pirâmides coloridas. Esse teste consiste em solicitar ao examinando que cubra o esquema utilizando-se dos quadrículos coloridos de modo a formar uma pirâmide bonita, conforme seu gosto. Nessa versão (VILLEMOR AMARAL, 1978) não se utiliza a construção das pirâmides feias introduzidas por Heiss e Hiltman (1951, apud VILLEMOR AMARAL, 1978). Cada encontro tinha um tempo médio de duração de uma hora e meia, havendo variações conforme o estado do paciente e suas possibilidades de colaborar.

O grupo normativo foi composto por indivíduos que nunca procuraram atendimento psicológico ou psiquiátrico e que residiam na mesma região dos pacientes. Para esse grupo manteve-se a proporção na distribuição conforme sexo, idade e escolaridade do grupo de pacientes. 
Os resultados foram analisados estatisticamente considerando-se a freqüência das cores e o aspecto formal das pirâmides.

\section{Resultados}

O teste de Pfister proporciona inúmeras variáveis relacionadas com a freqüência das cores e o aspecto formal que configura cada pirâmide. Para a análise da freqüência das cores considerou-se as dez cores que compõem o teste cujos valores podem variar de 0 a 100 , dependendo da porcentagem com que foram utilizadas. Além disso, consideramos as cores denominadas de constância absoluta, ou seja, que apareciam na composição das três pirâmides executadas.

O segundo conjunto de dados refere-se ao aspecto formal que é classificado conforme nove categorias distintas cada uma delas assumindo valores de um ou zero indicando presença ou ausência, para cada pirâmide construída.

A estratégia de análise empregada foi verificar quais variáveis seriam capazes de prever a pertença do indivíduo ao grupo de esquizofrênicos utilizando-se para isso da regressão logística. A regressão logística procura estimar a probabilidade de um indivíduo pertencer a determinado grupo confrontando o desempenho entre os grupos comparados.

Na Tabela 1 encontram-se os indicadores que foram significativos para distinguir o grupo de pacientes.

\section{TABela 1. RESUltados da ANÁLISE POR REGRESSÃo lOGíSTICA}

\begin{tabular}{lccccc}
\hline $\begin{array}{l}\text { Variáveis } \\
\text { do Pfister }\end{array}$ & $B$ & $E P$ & Wald & Sig. & Exp(B) \\
\hline CA_Ma e Vm &, 709 &, 440 & 2,599 &, 107 & 2,032 \\
Formação em camada Multicromática &,- 516 &, 716 &, 519 &, 471 &, 597 \\
Tapetes furados e desequilibrados &, 355 &, 616 &, 332 &, 564 & 1,426
\end{tabular}

Conforme a Tabela 1 verificamos que não foram encontradas cores cuja maior ou menor frequiência pudessem ser consideradas típicas para o grupo de esquizofrênicos. Por outro lado, ao considerarmos as cores de freqüência absoluta, observamos que vermelho e marrom são freqüentemente utilizados nas três pirâmides executadas pelos pacientes, e que o mesmo não ocorre com o grupo de não-pacientes.

Além disso, constatamos que a incidência de tapetes furados e desequilibrados é significativamente maior entre os pacientes enquanto a ocorrência de formações estratificadas é significativamente mais baixa nesse mesmo grupo quando comparados ao grupo de não-pacientes.

Seguindo com a análise, verificamos qual a probabilidade de acerto nos diagnósticos quando apoiados nesses indicadores. 


\section{Tabela 2. Coordenadas da Curva ROC}

\begin{tabular}{ccc}
\hline $\begin{array}{c}\text { Diagnóstico } \\
\text { positivo se a } \\
\text { probabilidade for } \\
\text { igual ou maior que }\end{array}$ & $\begin{array}{c}\text { Sensibilidade } \\
\text { (Verdadeiros } \\
\text { Positivos) }\end{array}$ & $\begin{array}{c}1-\text { Especificidade } \\
\text { (Falsos Positivos) }\end{array}$ \\
\hline, 0000000 & 1,000 & 1,000 \\
, 0729665 &, 900 &, 658 \\
, 0915750 &, 850 &, 622 \\
, 1074849 &, 750 &, 486 \\
, 1256163 &, 700 &, 432 \\
, 1465494 &, 600 &, 369 \\
, 1699234 &, 600 &, 351 \\
, 2106535 &, 550 &, 288 \\
, 2751546 &, 250 &, 045 \\
, 3505777 &, 200 &, 036
\end{tabular}

\section{ROC Curve}

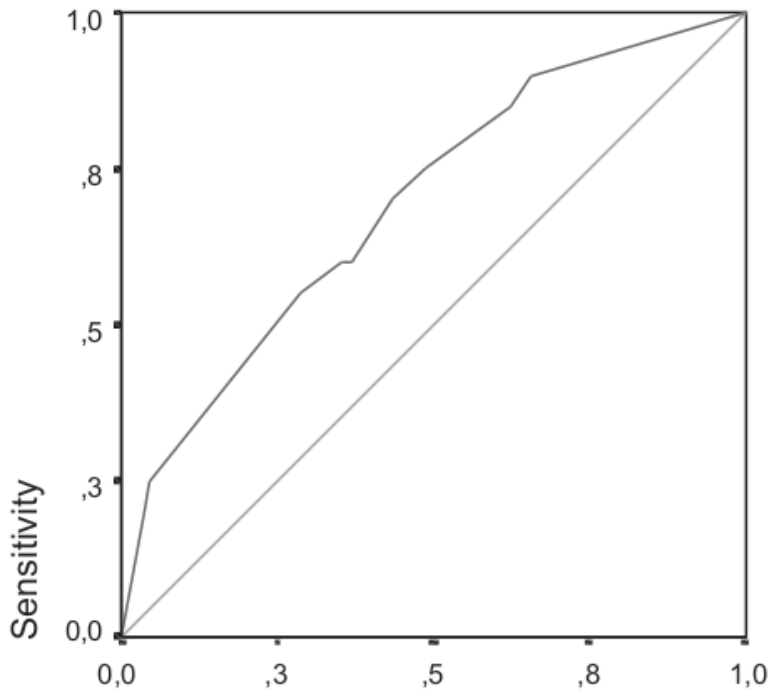

\section{1 - Specificity}

Diagonal segments are produced by ties. 
Verificamos que as coordenadas de ROC sugerem a probabilidade de diagnosticar corretamente $70 \%$ dos esquizofrênicos com base nesses indicadores, havendo entretanto um risco de $43 \%$ de falsos positivos entre o grupo de não-pacientes.

\section{Discussão}

O teste das Pirâmides Coloridas de Pfister é um instrumento cuja execução exige noções espaciais, apreensão de informações, escolha e combinação de cores e tonalidades, a fim de unificar partes e construir um todo. Essas associações cognitivas revelam quais são os recursos e mecanismos de defesa disponíveis e usados pelo examinando para elaborar aspectos da vida relacionados à esfera emocional. Ao executar o teste, alguns indivíduos respeitam a idéia de tridimensionalidade, outros percebem a tridimensionalidade no decorrer da execução e há ainda aqueles que ignoram a informação de origem posicionando as cores de forma tão irregular e desarmônica que o trabalho parece uma colcha de retalhos ou tapete. A colcha de retalhos é resultante de um modo de organização simplista, sem tantas exigências cognitivas, o que dá a impressão de que o examinando realizou a tarefa de forma aleatória, sem muita elaboração e atenção. As cores e tonalidades colocadas em desarmonia deixam a produção contrastante, pesadas e descontínuas e quando isso ocorre associado à presença do branco aplicado isoladamente ou em pequenos grupos, tem-se a impressão de furos. $\mathrm{O}$ fato de os pacientes terem apresentado esse aspecto formal em maior freqüência vai de encontro ao funcionamento cindido e com as características de dissociação no curso do pensamento, fragmentação, pouca coesão e desagregação da estrutura da personalidade. Seja pelo desequilíbrio observado na distribuição das cores, seja pelo aspecto interrompido dado pelo uso aleatório do branco, estamos diante da representação do desequilíbrio e fragmentação característicos do universo mental desses pacientes. Outra variável que foi significativa para diferenciar os pacientes esquizofrênicos de não pacientes foi o rebaixamento de pirâmides estratificadas em camadas multicromáticas que, segundo Villemor Amaral (1978) já é uma construção mais elaborada. Encontradas com maior freqüência em adultos normais, como também em crianças e adolescentes, tais pirâmides relacionam-se com a imaturidade ou com perturbações neuróticas.

No que diz respeito à freqüência das cores, nesse grupo investigado não encontramos o esperado aumento da cor branca anunciado na literatura consultada (JUSTO; VAN KOLCK,1976. HEISS; HALDER, 1983. VILLEMOR AMARAL, 1978) nem tampouco sua diminuição, conforme Carnio e Loureiro (1993), não havendo diferença significativa no uso dessa cor entre pacientes e não-pacientes.

Em nossas análises consideramos as cores de constância absoluta seguindo a indicação de Villemor Amaral (1978, p. 135) quando afirma que "é de suma importância para o estudo comparativo da fórmula cromática em função dos elementos cromáticos encarregados de sua estruturação". Assim encontramos a cor vermelha como cor constante na produção de esquizofrênicos, ainda que seus 
valores percentuais não tenham sido significativamente elevados. Se temos a predominância de tapetes furados e desequilibrados, a presença de vermelho nessas configurações pode reforçar a conotação de impulsos em estruturas enfraquecidas e conseqüente incontinência de reações, que indicam dificuldades de adaptação à realidade. Por outro lado, convém lembrar que a tonalidade preferida do vermelho, entre os que apresentaram essa cor com constância absoluta, foi o vermelho um, tonalidade mais esbranquiçada (cor-de-rosa), o que denota hipersensibilidade e labilidade (VILLEMOR AMARAL, 1978).

Acompanhando o vermelho, encontramos igualmente a constância absoluta da cor marrom. Em seu capítulo sobre as cores por duplas, Villemor Amaral (1978) afirma que vermelho e marrom constituem uma dupla cujo aumento concomitante ou sua combinação nas pirâmides, tem significado negativo de regressão, impulsividade sobretudo quando se apresentam em pirâmides desordenadas.

Seguindo a sugestão de Carnio e Loureiro, verificamos a ocorrência da Síndrome de Adaptação ao Ambiente - SAA - cujas componentes verde, azul e amarelo, podem significar determinados mecanismos de defesas, dependendo do modo como aparecem nas pirâmides. Entretanto, em nosso estudo, apenas 25\% dos pacientes apresentaram as combinações típicas de afastamento, as quais apareceram em $45 \%$ do grupo de 20 esquizofrênicos investigados por estas autoras.

\section{Conclusão}

Para o grupo de esquizofrênicos foi confirmado o aumento de tapetes furados e desequilibrados conforme o esperado a partir da literatura disponível. Já o aumento de branco não foi significativamente maior na população estudada quando comparado com as frequiências obtidas no grupo normativo. A associação de tapetes com a constância absoluta da cor vermelha, sobretudo em sua tonalidade mais clara, e com a constância absoluta da cor marrom, sugere a hipersensibilidade e incontinência de reações a que os pacientes estão sujeitos. Essas características são compatíveis com as descrições teóricas a respeito da esquizofrenia. Por outro lado, os indicadores de sensibilidade $-70 \%$ - e de especificidade $-43 \%$ obtidos para esse grupo, revelam que o teste de Pfister pode contribuir para o diagnóstico da esquizofrenia, mas deve ser associado a outros recursos diagnósticos até que futuros estudos venham ampliar sua validade para diagnóstico diferencial em psicopatologia.

\section{REFERÊNCIAS}

AMERICAN PSYCHIATRIC ASSOCIATION - APA- Manual Diagnóstico e Estatístico de Transtornos Mentais- DSM - IV. Porto Alegre: Artes Médicas, 1995.

CARNIO, E. C.; LOUREIRO, S.R. Caracterizações da percepção real de pacientes esquizofrênicos avaliados através das técnicas das Pirâmides Coloridas de Pfister. Revista Psico, Porto Alegre, v. 24, n. 1, p. 35-47, 1994. 
FIRST, M. B.; SPITZER, R. L.; GIBBON, M; WILLIAMS, J. B. W. Entrevista Clínica Estruturada para o DSM-IV Transtornos do Eixo I - SCID-I. Tradução: DEL BEN, C. M.; ZUARDI, A. W.; VILELA, J. A. A.; CEIPPA, J. A. S. New York: New York State Psychiatric Institute, 1996.

GABBARD, Glen O. Psiquiatria Psicodinâmica: baseado no DSM-IV. Porto Alegre: ArtMed, 1998.

HEISS, R.; HALDER, P. O teste das pirâmides de cores. São Paulo: Vetor, 1983. JUSTO, H.; VAN KOLCK, T. O teste das pirâmides de Cores. São Paulo: Vetor, 1976. KLEIN M. Notas Sobre a Observação do Comportamento dos Bebês, In: Os Progressos da Psicanálise. Rio de Janeiro: Guanabara Koogan, 1982. . Notas Sobre Alguns Mecanismos Esquizóides. In: . Os Progressos da Psicanálise. Rio de Janeiro: Guanabara Koogan, 1982.

PAIM, I. Esquizofrenia. São Paulo: EPU, 1990.

VILLEMOR AMARAL, F. Pirâmides Coloridas de Pfister. Rio de Janeiro: CEPA, 1978.

Recebido em: novembro/2004 Aceito em: maio/2005 